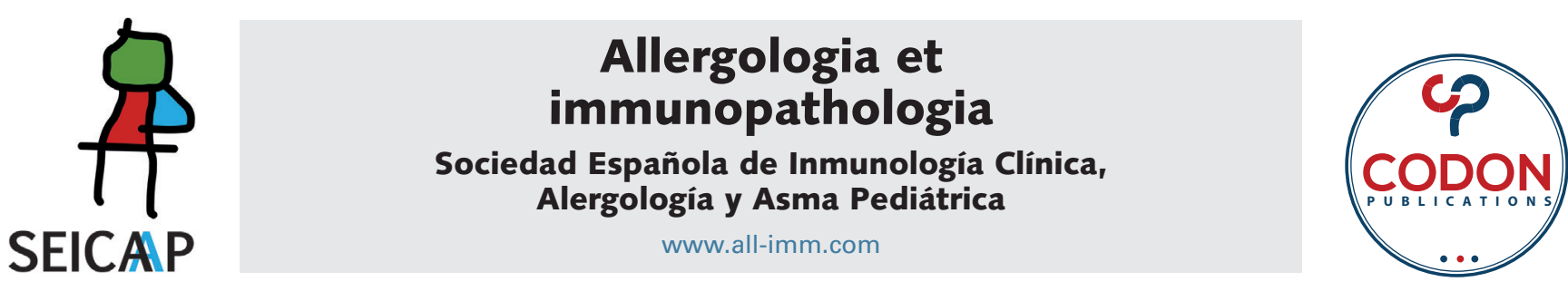

\title{
Specific IgE levels as an outcome predictor in egg-allergic children
}

\section{Jesús Rodríguez-Catalán*, Alba Manasa González-Arias, Almudena Vallespin Casas, Genoveva Del Río Camacho}

Pediatrics Department, Hospital Universitario Fundación Jiménez Díaz, Madrid, Spain

Received 3 April 2020; Accepted 13 September 2020

Available online 2 January 2020

\section{KEYWORDS \\ egg allergy; \\ IgE levels; oral food challenge; cut-off value; children; positive predictive value}

\begin{abstract}
Background: Diagnosis of immunoglobulin E (IgE)-mediated egg allergy is often based on both a compatible clinical history and either elevated IgE levels or a positive skin prick test. However, the gold standard is the oral food challenge (OFC). Previous studies have pointed to a correlation between IgE levels and OFC outcomes.

Objective: This study aimed to determine the relationship between IgE levels and the outcome of OFC, seeking to establish cut-off OFC values that indicate a high likelihood of positive OFC results.

Methods: A total of 198 patients who underwent OFC (and a serological IgE antibody assay within the three preceding months) were reviewed and divided by OFC type (i.e., baked, cooked, or raw egg). IgE-specific levels were assessed against the challenge outcome as well as cut-offs proposed by other authors.

Results: Receiver-operating characteristic (ROC) curve analysis yielded a potentially useful ovomucoid IgE-specific cutoff used in OFC with cooked egg and several egg white and ovalbumin IgE-specific cut-offs for OFC with raw egg. We found no significant relationship between other specific IgE concentrations and the challenge threshold level with baked eggs.

Conclusions: IgE-specific concentrations are useful as predictors of OFC outcome and should be considered when selecting patients challenge testing with boiled or raw egg. However, patients should undergo OFC with baked egg regardless of IgE levels.
\end{abstract}

(c) 2021 Codon Publications. Published by Codon Publications.

*Corresponding author: Jesús Rodríguez Catalán. Pediatrics Department, Hospital Universitario Fundación Jiménez Díaz Avenida Reyes Católicos 2, 28040 Madrid, Spain. Email address: jesusrodricatalan@gmail.com 


\section{Introduction}

Egg-protein allergy is the most common allergy in the first 2 years of life (incidence of $2.4-2.6 \%) .{ }^{1,2}$ It is usually immunoglobulin $\mathrm{E}$ (lgE)-mediated ${ }^{2}$ and more prevalent in patients with atopic dermatitis and/or cow's milk allergy. ${ }^{1}$ Most egg-protein allergens have been described in egg white, ${ }^{1}$ ovalbumin (OVA, Gal d2) being the most abundant, followed by ovomucoid (OVM, Gal d1), ovotransferrin/conalbumin (Gal d3), and lysozyme (Gal d4). OVM and OVA differ mainly in their chemical properties, as OVM maintains its immunogenicity for 20 min after being cooked, while OVA becomes denatured under heat exposure. ${ }^{2}$ Consequently, patients sensitized only to OVA usually react only to lesscooked forms of the egg while patients sensitized to OVM may experience reactions with egg in any state.

Allergic reactions to egg vary widely and diagnosis is based on a suggestive medical history followed by proof of the IgE-related mechanism (prick test and/or serum specific- IgE levels). ${ }^{1-3}$ Although oral food challenge (OFC) remains the gold standard test, clinicians may omit this procedure in cases of a highly suggestive clinical history and positive specific IgE or prick test, to avoid unnecessary reactions. When undertaken, OFC may also give prognostic information about the risk of severe reactions. ${ }^{4}$

Following diagnosis, patients are reexamined periodically by repeat determination of specific IgE. Patients with a decrease in IgE and no dietary transgressions in the preceding months may be recommended to undergo an OFC to confirm the persistence or resolution of the allergy, ${ }^{2}$ often leading to a favorable prognosis in which the disease resolves before 6 years of age. ${ }^{2}$ However, $15-20 \%$ of children will remain allergic, tending to have poorer adherence to exclusion diets with time, more severe allergic reactions, and a significantly detrimental impact on the quality of life. ${ }^{1}$

As a drop in specific-lgE levels may predict tolerance, ${ }^{3,5,6}$ some studies have proposed particular specific-IgE values as cut-off points with high positive predictive value (PPV), ${ }^{1-3,6}$ while other authors have attempted to define a threshold value applicable to skin tests or specific-lgE levels to predict tolerance. ${ }^{3-5}$ However, these cut-off points vary according to several factors, such as how much the egg has been cooked, the type of allergen used in skin testing, patient age, and disease severity.

Based on this existing evidence, we hypothesized that a cut-off value for egg-specific IgE exists, above which all OFCs will be positive. The use of cut-off levels enables more rational use of resources and can avoid subjecting patients with persistent allergy to potential unnecessary adverse events.

\section{Materials and methods}

To conduct this longitudinal and retrospective study, we revised all the OFCs with egg in children between 2012 and 2018. All were previously diagnosed with IgE-mediated egg allergy and had had a blood test within the three previous months. We excluded all non- IgE-mediated allergies and children diagnosed by prick tests only. The study was approved by the research ethics committee of the hospital. Informed consent was required in all cases before the OFC.
In addition to general patient information (i.e., OFC date, age at diagnosis and at OFC, sex), we also recorded the following data: symptoms; specific IgE levels to egg white, yolk, OVA, OVM, and whole egg (in kU/L); comorbidities (i.e., asthma, atopic dermatitis, other food allergies); and the form of egg used in the OFC (boiled, raw, omelet, baked goods). We additionally gathered data related to the OFC such as positive/negative outcomes and resolution of the allergy (or not). In the positive cases, we noted the symptoms (i.e., respiratory, cutaneous, gastrointestinal, or anaphylaxis) and medication needed (none, antihistamine, corticosteroid, adrenaline, or bronchodilator).

All the patients included had received egg-specific IgE determinations upon diagnosis and in the 3 months prior to the OFC, both using the ImmunoCAP autoanalyzer (Phadia Laboratory Systems, Uppsala, Sweden), a system that detects IgE concentrations within the range of $0 \mathrm{kU} / \mathrm{L}$ to $100 \mathrm{kU} / \mathrm{L}$. Specific-IgE titers equal to or higher than 0.35 $\mathrm{kU} / \mathrm{L}$ are widely accepted as positive. ${ }^{7}$

The form of egg used to challenge each patient (boiled egg white, raw egg white, or baked goods) was chosen by the physician in charge, considering the form that caused symptoms and the patient's specific-IgE levels. The sensitized children with no prior reported exposition were challenged with boiled egg unless parents preferred to start with baked goods. Oral food challenge with baked goods (containing both, egg white and yolk) has no standardized protocol in our hospital, and dose levels are determined based on the percentage of egg in the recipe or product brought by the patient. Similarly, when using a boiled egg, it is provided by the hospital kitchen, but cooked following their usual practice although it has been reported egg should be cooked for 20 min to reduce its allergenicity. ${ }^{2}$

Patients underwent an OFC with boiled or raw egg white, as it was assumed that patients who tolerated egg white would also tolerate yolk, as the latter contains far fewer proteins. The doses used for boiled egg white were $1 / 12,1 / 6$, and $1 / 4$ of the egg with 30 min separating each intake until a maximum of half an egg white was reached. For raw egg white, the amounts administered were 0.1, 0.9, and $4 \mathrm{~mL}$, also with a 30-min wait between each dose, until patients had ingested a total of $5 \mathrm{~mL}$ of the $30 \mathrm{~mL}$ volume of a whole egg white.

Challenges were considered positive if the patient developed symptoms (cutaneous, respiratory, gastrointestinal, or anaphylaxis) and negative when the child tolerated the egg properly. Patients were kept under observation for $2 \mathrm{~h}$ after the last dose. All patients were followed up by the physicians after the OFC, reporting late events; when those effects happened within the first week after the challenge, the patient was told to avoid the food, as the OFC was assumed positive. When reactions happened later, the treatment was adjusted individually. These very late reactions have not been registered for this study.

Statistical analysis was performed using the IBM SPSS Statistics program, version 25, using Chi-square and MannWhitney U-tests. NCSS 2020 was utilized to construct receiver operating characteristics (ROC) curves of the sensitivity, specificity, and predictive values of the different specific-IgE points. The curve represents the pairs (sensitivity and 1-specificity or false positive rate) obtained from all possible cut-off values in the test. ${ }^{8}$ Therefore, values 


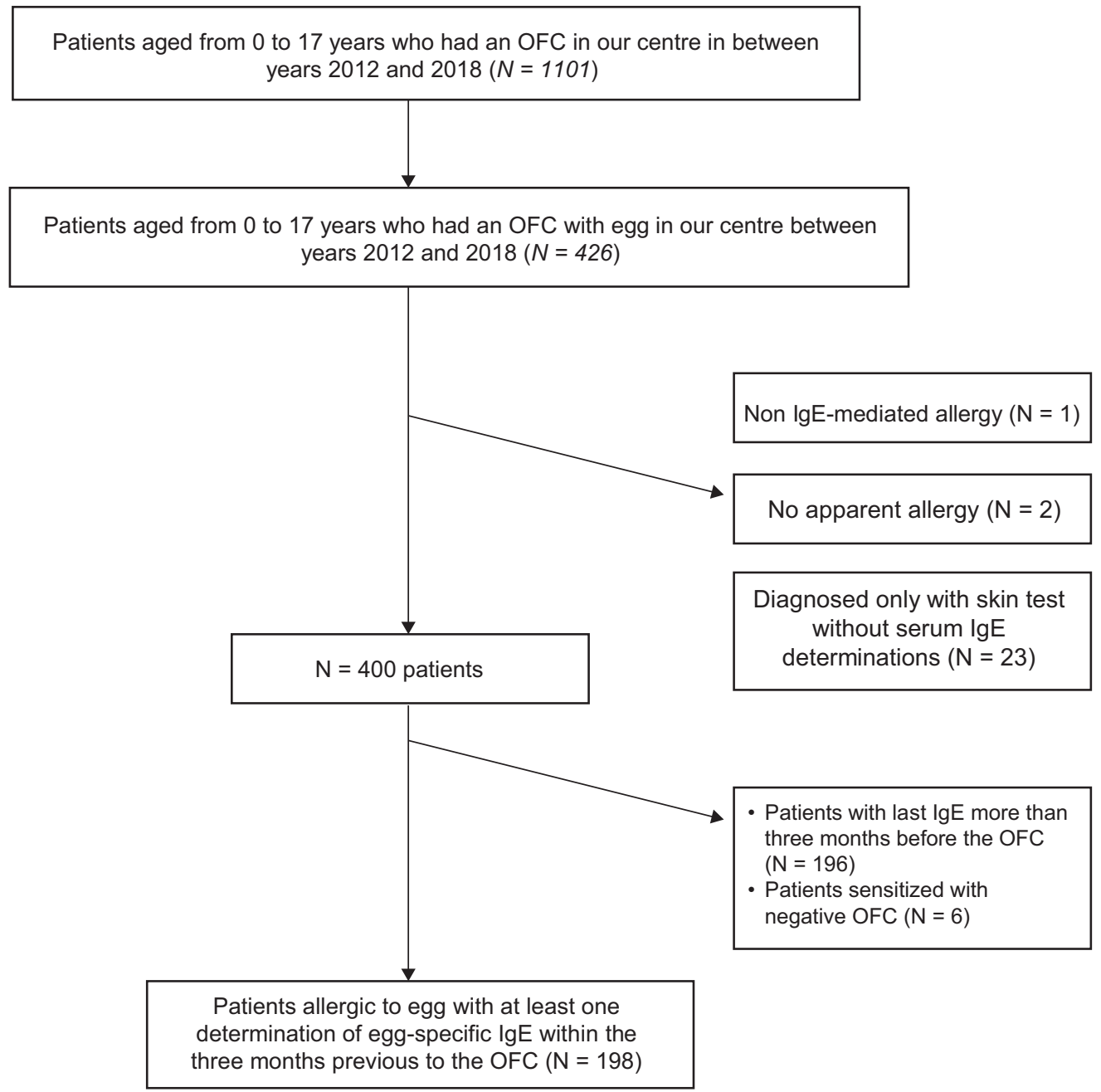

Figure 1 Flowchart showing patients selected for this study. OFC: oral food challenge.

above or below a given threshold can be diagnosed as positive and negative, respectively. As specificity increases, sensitivity decreases and vice versa. The area under the curve (AUC) was estimated using the trapezoidal method with a confidence interval $(\mathrm{Cl})$ of $95 \%$. $P$ values $<0.05$ were considered statistically significant.

\section{Results}

We reviewed a total of 1101 challenges between 2012 and 2018 (Figure 1), 426 of which involved egg. Of these, 26 patients were excluded: one had non-IgE mediated allergy, two had no apparent allergy as indicated by a negative skin prick test or specific IgE with low clinical suspicion (although parents requested to undergo the test in the hospital because they did not want to reintroduce egg at home out of fear of reactions), and 23 patients' diagnoses had relied on skin tests exclusively. Of the remaining 400, 196 were also excluded since more than 3 months had elapsed between their most recent IgE measurement and the challenge. From the remaining 204 children, 41 were sensitized to egg found in the course of IgE determinations for other allergies, with only those who had a positive OFC with egg afterwards being included. Thus, the final sample included 198 challenges.

The demographic characteristics of the population studied are summarized in Table 1. The median age at diagnosis was 12 months, and 128 patients (64.64\%) were male. Skin symptoms were the most common at diagnosis $(87.03 \%)$, followed by gastrointestinal manifestations (20.98\%) and, lastly, respiratory problems (5.55\%); with anaphylaxis in $4.32 \%$ of the patients. Of the patients studied, $17.68 \%$ had never eaten egg and were initially diagnosed as sensitized to the egg in the course of IgE determinations for other food allergies, but had a positive OFC later that confirmed the allergy. The concomitant allergic disease was a common finding, including atopic dermatitis (61.61\%), wheezing or asthma (30.30\%), co-sensitization to cow's milk $(31.81 \%)$, and other food allergies (34.84\%).

Considering all challenges included in the final sample, the median age at the OFC was 18 months, and $29.29 \%$ had a positive challenge. Positive results were mainly indicated by swelling or skin signs $(\mathbf{7 7 . 1 9 \% )}$ followed by more severe reactions (affecting two or more systems) in $15.80 \%$ of the patients, none of these reactions was fatal nor required 
Table 1 Demographic characteristics of the studied population.

\begin{tabular}{|c|c|c|}
\hline Characteristics & & Total $(n=198)$ \\
\hline Sex & M/Total (male \%) & $128 / 198(64.64)$ \\
\hline \multirow[t]{2}{*}{ Median age (months) at diagnosis } & Mean (standard deviation) & $12.56(6.80)$ \\
\hline & Median (interquartile range) & $12.00(10-13.00)$ \\
\hline \multicolumn{3}{|l|}{ Egg allergy suspected because of: } \\
\hline Symptoms after eating egg & N (\%) & $162 / 198(81.81)$ \\
\hline Cutaneous & N (\%) & $141 / 162(87.03)$ \\
\hline Gastro-intestinal & N (\%) & $34 / 162(20.98)$ \\
\hline Respiratory & N (\%) & 9/162 (5.55) \\
\hline Anaphylaxis & N (\%) & $7 / 162(4.32)$ \\
\hline Sensitization & N (\%) & $36 / 198(18.18)$ \\
\hline History of atopic dermatitis & $\mathrm{N}(\%)$ & $122 / 198(61.61)$ \\
\hline History of asthma or wheezing & N (\%) & $60 / 198(30.30)$ \\
\hline History of cow's milk allergy & $\mathrm{N}(\%)$ & 63/198 (31.81) \\
\hline History of other food allergies & N (\%) & 69/198 (34.84) \\
\hline \multicolumn{3}{|l|}{ Specific-IgE values at diagnosis } \\
\hline Yolk IgE & Median (interquartile range) & $0.38(0.09-0.89)$ \\
\hline Egg-white IgE & Median (interquartile range) & $2.00(0.66-5.62)$ \\
\hline Ovalbumin IgE & Median (interquartile range) & $1.29(0.43-5.18)$ \\
\hline Ovomucoid IgE & Median (interquartile range) & $0.64(0.02-3.30)$ \\
\hline Egg lgE & Median (interquartile range) & $1.74(0.58-4.86)$ \\
\hline
\end{tabular}

Regarding sex, the percentage represents the males as a proportion of the total sample. For categorical variables, the table indicates the percentage of patients who exhibited these variables compared to the total; results for quantitative variables are expressed as median and interquartile range.

intensive care unit admission. The remaining $7.01 \%$ had respiratory or digestive manifestations.

Table 2 shows median egg-specific IgE concentrations from the 47 OFCs performed with baked goods, divided into positive and negative results. None of the comparisons made between the two groups reached statistical significance.

Of the 106 OFCs that used boiled egg white, patients with a negative result had a median level of ovomucoid IgE of $0.02 \mathrm{kU} / \mathrm{L}$ (interquartile range, 0.00-0.42) as compared to those children with a positive challenge, who had median levels of $0.55 \mathrm{kUA} / \mathrm{L}$ (0.17- 1.75), $P<0.01$ (Table 2). On the contrary, no differences were found between the levels of ovalbumin in both groups.

The 43 challenges performed with raw egg white revealed a median of $0.96 \mathrm{kUA} / \mathrm{L}(0.62-3.70)$ for positive OFCs, while children with negative test results had median values of $\mathrm{kUA} / \mathrm{L}(0.12-0.92), P<0.01$. Also, ovalbumin-lgE and yolk-lgE values were higher $(P<0.01)$ in the positive OFC group. We found no differences when comparing the levels of OVM (Table 2).

Two patients were challenged using omelet and were included in the overall descriptive analysis, but no comparative analysis was performed, as this was such a small group.

For each of the variables that reached statistical significance, a figure is shown with the corresponding ROC curve along with the AUC and the $95 \% \mathrm{CI}$. The cut-off values were determined according to previous studies and the specificity, sensitivity, and predictive values were found based on the ROC curves. The only curve with good discriminative capacity for the boiled egg was the one constructed for ovomucoid-specific IgE (Figure 2), for which the AUC was 0.756 and the $95 \% \mathrm{Cl}$ was $0.63-0.83$.
Also reflected in Figure 2, when analyzing the OFC with raw egg white, curves with good differentiating capacity were found for specific IgE to egg white, yolk, and OVA. For egg-white IgE, the AUC was 0.802 with a $95 \% \mathrm{Cl}(0.57-0.91)$, while the AUC for yolk- specific IgE was $0.816(0.59-0.92)$, and for OVA, 0.871 (0.66-0.95).

Table 3 includes the possible cut-off points obtained from ROC curves, thus indicating the proposed specific-lgE levels to identify those patients with $>70 \%,>90 \%$, and $100 \%$ probabilities of having a positive OFC.

For challenges with boiled egg, we failed to find values with a good PPV; the highest PPV was $58.33 \%$, corresponding to an ovomucoid-IgE value of $1.73 \mathrm{kU} / \mathrm{L}$ ( $93.15 \%$ specificity).

With the raw egg white, a patient with a specific IgE of $2.74 \mathrm{kU} / \mathrm{L}$ is likely to have an $85.71 \%$ probability of having a positive OFC; this likelihood rises to $100 \%$ if the level is above $4.57 \mathrm{kU} / \mathrm{L}$. We propose the cut-off level of 2.98 $\mathrm{kU} / \mathrm{L}$ for ovalbumin-IgE, also with a PPV of $100 \%$. These two cut-off values had a specificity of $100 \%$. We decided not to consider yolk-specific IgE due to its low relevance in daily clinical practice (being considered less allergenic because it has more lipids and fewer proteins than egg white) and its absence from the literature reviewed for the present study.

\section{Discussion}

Several articles have proposed that high specific-IgE concentrations may be used to predict clinical symptoms, making it worthwhile to establish cut-off points based on predictive values to inform decisions on whether or not to perform an OFC. ${ }^{9-13}$ Here, we aimed to find the relation 
Table 2 Percentage of positive challenges, median age, and median of specific IgE for each OFC (with baked goods, boiled egg white, or raw egg white).

\begin{tabular}{|c|c|c|c|c|c|}
\hline & $\begin{array}{l}\text { Median age in } \\
\text { months (range) }\end{array}$ & $\begin{array}{l}\text { Positive } \\
\text { OFC (\%) }\end{array}$ & $\begin{array}{l}\text { Median of IgE in negative } \\
\text { OFC (Interquartile range) }\end{array}$ & $\begin{array}{l}\text { Median of IgE in positive } \\
\text { OFC (Interquartile range) }\end{array}$ & $\mathrm{P}^{* *}$ \\
\hline Global $(n=198)^{*}$ & $\begin{array}{c}18.00 \\
(12.00-31.00)\end{array}$ & $29.29 \%$ & & & \\
\hline Baked goods $(n=47)$ & $\begin{array}{c}14.00 \\
(12.00-26.00)\end{array}$ & $27.65 \%$ & & & \\
\hline Egg-white IgE & & & $3.44(1.55-7.00)$ & $2.76(1.78-14.95)$ & NSS \\
\hline Yolk lgE & & & $0.37(0.17-1.50)$ & $0.33(0.13-3.18)$ & NSS \\
\hline Ovalbumin IgE & & & $2.10(0.44-4.69)$ & $1.23(0.33-11.49)$ & NSS \\
\hline Ovomucoid lgE & & & 3.21 (1.30-5.97) & $3.42(2.02-9.47)$ & NSS \\
\hline Boiled egg $(n=106)$ & $\begin{array}{c}18.00 \\
(12.00-28.00)\end{array}$ & $24.52 \%$ & & & \\
\hline Egg-white IgE & & & $0.87(0.27-2.77)$ & $0.91(0.32-1.88)$ & NSS \\
\hline Yolk lgE & & & $0.20(0.05-0.68)$ & $0.14(0.06-0.38)$ & NSS \\
\hline Ovalbumin IgE & & & $0.74(0.23-2.34)$ & $0.44(0.10-1.52)$ & NSS \\
\hline Ovomucoid IgE & & & $0.02(0.00-0.42)$ & $0.55(0.17-1.75)$ & $<0.01$ \\
\hline Raw egg White $(n=43)$ & $\begin{array}{c}26.00 \\
(14.00-36.00)\end{array}$ & $44.18 \%$ & & & \\
\hline Egg-white IgE & & & $0.32(0.12-0.92)$ & $0.96(0.62-3.70)$ & $<0.01$ \\
\hline Yolk IgE & & & $0.11(0.04-0.29)$ & $0.52(0.19-1.25)$ & $<0.01$ \\
\hline Ovalbumin IgE & & & $0.30(0.05-0.75)$ & $0.93(0.72-3.18)$ & $<0.01$ \\
\hline Ovomucoid IgE & & & $0.06(0.01-0.20)$ & $0.12(0.02-0.60)$ & NSS \\
\hline
\end{tabular}

*Two patients have been excluded after this analysis because their OFC was performed using an omelet; this group was not sufficiently large to perform a comparative analysis.

** $\mathrm{P}$ value obtained after comparing median IgE values between positive and negative OFC results. OFC: oral food challenge; NSS: not statistically significant.
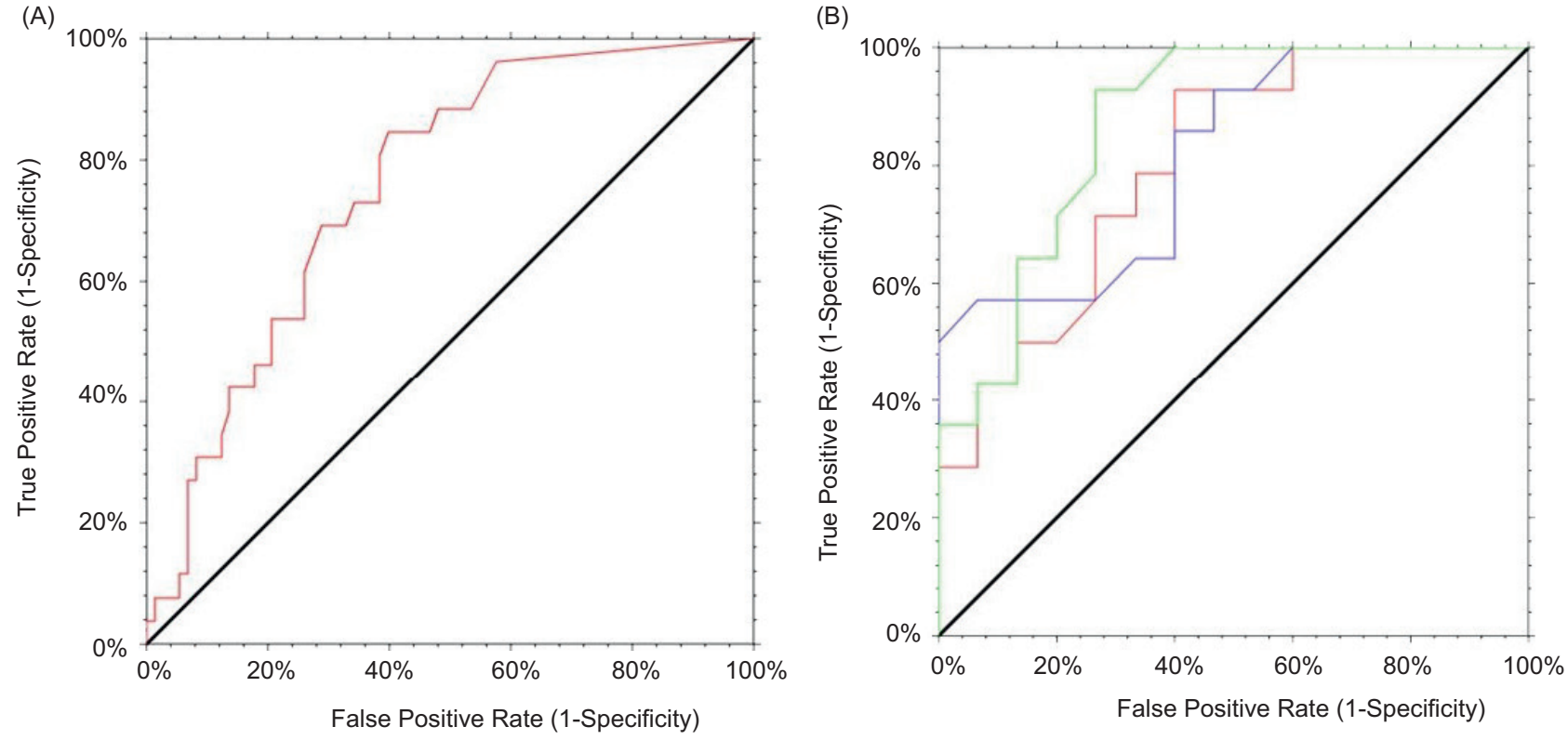

Figure 2 ROC curves obtained from OFC with egg. In the ordinate sensitivity is shown, and in the abscissa the false positive rate (1-specificity) is reflected. (A) OFC with boiled egg (ovomucoid IgE levels). AUC $=0.756,95 \% \mathrm{Cl}$ : 0.63- 0.83. (B) OFC with raw egg: Egg-white IgE values in red. AUC = 0.802, 95\% Cl: 0.57-0.91; Yolk IgE values in blue. AUC = 0.816, 95\% Cl: 0.59-0.92; Ovalbumin IgE values in green. AUC = 0.871, 95\% Cl: 0.66-0.95; ROC: Receiver-operating characteristic; OFC: Oral food challenge; AUC: Area under the curve; $\mathrm{Cl}$ : confidence interval. 
Table 3 Cut-off points set to reach positive predictive values (PPV) around 70, 85, and 100\%.

\begin{tabular}{|c|c|c|c|c|c|}
\hline & Cut-off point (kU/L) & Sensitivity (\%) & Specificity (\%) & PPV (\%) & NPV (\%) \\
\hline \multicolumn{6}{|l|}{ Boiled egg } \\
\hline Ovomucoid IgE & 1.73 & 26.92 & 93.15 & 58.33 & 78.16 \\
\hline \multicolumn{6}{|l|}{ Raw egg white } \\
\hline \multirow[t]{3}{*}{ Egg White IgE } & 0.59 & 92.86 & 66.00 & 68.42 & 90.00 \\
\hline & 2.74 & 42.86 & 93.33 & 85.71 & 63.74 \\
\hline & 4.57 & 28.57 & 100 & 100 & 60.00 \\
\hline \multirow[t]{3}{*}{ Yolk IgE } & 0.30 & 57.14 & 80.00 & 72.73 & 66.67 \\
\hline & 0.50 & 57.14 & 93.33 & 100 & 70.00 \\
\hline & 0.53 & 50.00 & 100 & 100 & 68.18 \\
\hline \multirow[t]{3}{*}{ Ovoalbumin IgE } & 0.38 & 100 & 60.00 & 70.00 & 100 \\
\hline & 2.44 & 42.86 & 93.33 & 85.71 & 63.64 \\
\hline & 2.98 & 35.71 & 100 & 100 & 62.50 \\
\hline
\end{tabular}

The highest PPV has been chosen for each value.

PPV: positive predictive value; NPV: negative predictive value.

between IgE levels and challenge outcomes, to determine the point above which an OFC is most likely to be positive, thus indicating that we may avoid performing the test, and vice versa.

\section{Baked goods}

None of the IgE values or ROC curves for OFC with baked goods reached statistical significance nor exhibit good discriminative capacity, so we decided not to include them. This failure to achieve acceptable results leads us to question whether there is any cut-off point with $100 \%$ accuracy for cases of allergy with successful challenge results for baked goods.

These results indicate that we may have a positive OFC despite low specific-IgE levels and vice versa. We can thus claim that these patients may always be challenged, as the outcome varies from child to child. Patients in this report were challenged using muffins or similar products; but recently, we have changed our protocols, and after a positive OFC, we re-challenge the patient with biscuit, with better tolerance in the majority of cases.

Some authors argue that baked egg added to the diet following a negative OFC is well tolerated and shortens the time required by the patient to reach tolerance to other forms of egg $^{14}$ when compared to those who have maintained an egg-free diet after being found to have high egg-specific IgE, without undergoing OFC. Another benefit of reintroducing products with baked egg is the improvement in the quality of life, allowing the child to eat a wider variety of food to fulfill their nutritional requirements. ${ }^{14}$ Nevertheless, the egg should be reintroduced under medical supervision, as there are no studies on the safety and efficacy of this method to date.

\section{Boiled egg}

As mentioned previously, ROC curves for OFC with boiled egg revealed that a maximum PPV of $58.33 \%$ for $1.73 \mathrm{kU} / \mathrm{L}$ for OVM-IgE, also with a specificity of $93.15 \%$. Studies of OFC with boiled egg are scarce, and their findings inconclusive. One article from $2008,{ }^{15}$ set extremely high IgE cutoff points (egg white: $7.4 \mathrm{kU} / \mathrm{L}$; OVM: $10.8 \mathrm{kU} / \mathrm{L}$ ), based on a highly atopic sample. Another study, in 2014, ${ }^{16}$ reported levels that more closely resemble those of our study (egg white: $4.08 \mathrm{kU} / \mathrm{L}$; OVA: $2.8 \mathrm{kU} / \mathrm{L}$; OVM: $3.74 \mathrm{kU} / \mathrm{L})$, although the patients were significantly older (median age 8.2 years). All the other publications we reviewed show no cut-off points for OVM, making this a potentially novel and valuable aspect of our work. As for OVA-IgE, we found no significant values, but hypothesize that the heat-sensitive nature of this protein means that these patients may tolerate boiled egg regardless of specific IgE levels.

\section{Raw egg white}

In OFCs with raw egg white, the AUC had a high discriminative capacity for specific IgE to egg white, yolk, and OVA (Figure 2). We, therefore, have included possible cut-off values (Table 3 ) to identify those patients who are likely to have a positive challenge.

Until recently, the utility of specific IgE levels was unknown. Sampson ${ }^{12}$ was one of the first researchers to propose predictive values for different allergens, reporting that IgE levels $>6 \mathrm{kU} / \mathrm{L}$ were linked to a positive OFC, a finding that had a $96 \%$ PPV. Subsequent studies, however, have reported highly different values; Boyano Martínez et al. ${ }^{10}$ observed that egg-white lgE concentrations $>0.35 \mathrm{kU} / \mathrm{L}$ indicated that an OFC would be positive, while the working group led by Osterballe ${ }^{13}$ found no clear positive threshold in specific IgE levels for OFC but defined $\operatorname{lgE}>1.5 \mathrm{kU} / \mathrm{L}$ to be reliable levels indicating a positive diagnosis. More recently, in a position paper released in 2013, Martorell et al. ${ }^{1}$ concluded that egg-white IgE levels $>1.7 \mathrm{kU} / \mathrm{L}$ in children under age 2 years (and $>7.3 \mathrm{kU} / \mathrm{L}$ among patients above that age) indicate a persistent allergy, thus predicting a positive OFC with raw egg white.

Our work bears certain similarities with Sampson's study $^{12}$ : they also performed OFC with egg white and 
proposed a cut-off point of $3.4 \mathrm{kU} / \mathrm{L}$ (82\% sensitivity, $84 \%$ specificity), with this value being slightly higher than the one we obtained. One factor that may have skewed their results is that their population had a high prevalence of atopic dermatitis, with a median total IgE of $3000 \mathrm{kU} / \mathrm{L}$ (much higher than the median total IgE in our study).

Boyano Martínez et al. ${ }^{10}$ set a cut-off value of $0.35 \mathrm{kU} / \mathrm{L}$, with specificity and sensitivity $<80 \%$. Their population was recruited from patients who had had a previous allergic reaction after eating an egg and a median egg-white IgE of $40 \mathrm{kU} / \mathrm{L}$. The sample had additional similarities to our children, although our values were slightly higher than theirs $(0.35 \mathrm{kU} / \mathrm{L})$, the reference value accepted as positive in most laboratories.

Regarding OVM, no statistically significant values have been found. However, as this protein is not sensitive to heat, ${ }^{1}$ measuring OVM levels alone may be more useful when planning an OFC with boiled egg (provided the other allergens may lose their allergenic power once heated), while challenges using raw egg must take more allergens into account.

One explanation posited for the substantial differences between cut-offs ${ }^{13}$ relates to differences between study populations. Using specificity when setting a cut-off point may help minimize the influence variations in allergy prevalence between populations since specificity is not susceptible to prevalence. ${ }^{12}$

Rather than representing all possible cut-off points and their specificity in table form, Table 3 contains our chosen points and their PPV. All the cut-offs we propose have high specificity, thereby enabling these values to be extrapolated to different populations. These cut-offs are, however, only helped to confirm allergy diagnosis when the symptoms are suggestive. OFC remains the gold-standard diagnostic test and, especially in cases of doubt, should be performed before prescribing an exclusion diet or reintroducing the offending food in the diet. ${ }^{12}$

Concomitant allergic disease and co-sensitization usually increase total levels of IgE in comparison with the non-atopic population, so proposed cut-off values in these patients will always be higher than in patients such as ours.

As a secondary objective, we tried to find any relation between these predisposing factors (i.e., atopic dermatitis, asthma/wheezing, other food allergies). Our analysis failed to reveal a significant relationship, so, the decision was made not to include this result due to its potential to distract from the main objective.

Finally, some may believe that these differences could be attributed to individual physicians' criteria, variations in techniques used, and the chemical reagents used in each laboratory to determine IgE. Although IgE is measured according to a standardized method with a universal protocol, minimum discrepancies may be found between manufacturers. ${ }^{7}$ Regarding differences liked in clinical practice, most of the studies are prospective and have performed serial OFC regardless of specific IgE levels. In our study, not all the patients have undergone OFC in light of their high specific-IgE levels and thus a high likelihood of a positive test, thereby opting to avoid exposing the patient to unnecessary risk.

In conclusion, depending on the state of the egg used in the OFC, this study has found a relation between specific IgE values to egg white, OVA, and OVM and the outcome of an OFC, enabling us to propose a series of cut-offs that may be useful in routine clinical practice:

- OFC with baked goods: no threshold values have been identified. Therefore, regardless of IgE levels, OFC can be performed to determine whether the patient tolerates baked egg and, if so, reintroduce the food into the diet, with a close medical follow-up.

- OFC with boiled egg: if OVM-IgE is $1.73 \mathrm{kU} / \mathrm{L}$ or higher, there is a $58.33 \%$ probability of a positive challenge (specificity of $93.15 \%$ ). We believe it is unnecessary to take into account the value of OVA-IgE, as it is heat-sensitive.

- OFC with raw egg white: egg-white IgE $2.74 \mathrm{kU} / \mathrm{L}$ and OVA-IgE $2.44 \mathrm{kU} / \mathrm{L}$ are our cut-off points, showing specificity of $93 \%$ and a PPV of $86 \%$. No significant values were found for OVM-IgE, although we would argue that these levels alone may be more useful in challenges with boiled egg (assuming that the other allergens may lose their allergenic power when heated) while using raw egg there are more active allergens to consider.

Further prospective multi-centered studies with larger sample sizes are needed to confirm and generalize the utility of our proposed cut-offs and to identify cut-off values for the rest of the egg (and other food) allergens.

However, OFC remains the best tool available until this or other values have been validated, especially to confirm the resolution of the allergy in patients with decreasing specific-IgE levels. One exception to this involves cases where the patient reports accidental dietary transgressions, without symptoms, in which the OFC may be avoidable.

\section{Limitations}

Our article is a retrospective study reflecting our daily clinical practice, where our goal is to achieve tolerance as soon as possible and to avoid exposing the patient to unnecessary risks. Moreover, since we had no funding for this study, we could not implement standardized cooking protocols.

Additionally, not all the patients underwent an OFC regardless of their specific IgE levels, as in other reports. Instead, children were challenged only when we thought they had overcome their allergy, as we assumed persistent allergy when the specific IgE remained high.

\section{Conflicts of interest}

The authors have no conflicts of interest to declare.

\section{Acknowledgments}

The authors thank Oliver Shaw for editorial assistance.

\section{References}

1. Matorell A, Alonso E, Echeverría L, López MC, Martin F, Nevot S, et al. Position document: IgE-mediated allergy to 
egg protein. Allergologia Immunopathologia. 2013;41:320-336. https://doi.org/10.1016/j.aller.2013.03.005.

2. García Ara MC. Alergia al huevo en el niño. Protocdiagn ter pediatr. 2013;1:37-50.

3. Calvani M, Arasi S, Bianchi A, Caimmi D, Cuomo B, Dondi A, et al. Is it possible to make a diagnosis of raw, heated, and baked egg allergy in children using cutoffs? A systematic review. Pediatr Allergy Immunol. 2015;26(6):509-521. https:// doi.org/10.1111/pai.12432

4. Furuya $K$, Nagao $M$, Sato $Y$, Ito $S$, Fujisawa T, IPAD3g investigators. Predictive values of egg-specific IgE by two commonly used assay systems for the diagnosis of egg allergy in young children: a prospective multicenter study. Allergy. 2016;71(10):1435-1443. https://doi.org/10.1111/all.12912

5. Gradman J, Mortz CG, Eller E, Bindslev-Jensen C. Relationship between specific IgE to eff components and natural history of egg allergy in Danish children. Pediatr Allergy Immunol. 2016;27(8):825-830. https://doi.org/10.1111/pai.12644

6. Celik-Bilgili S, Mehl A, Verstege A, Staden U, Nocon M, Beyer $\mathrm{K}$, et al. The predictive value of specific immunoglobulin $E$ levels in serum for the outcome of oral food challenges. Clin Exp Allergy. 2005;35(3):268-273. https://doi. org/10.1111/j.1365-2222.2005.02150.x

7. Park KH, Lee J, Sim DW, Lee SC. Comparison of Singleplex specific IgE detection immunoassays: ImmunoCAP Phadia 250 and Immulite 2000 3gAllergy. Ann Lab Med. 2018;38(1):23-31. https://doi.org/10.3343/alm.2018.38.1.23

8. Cerda J, Cifuentes L. Uso de curvas ROC en investigación clínica: Aspectos teórico-prácticos. Rev Chil Infect. 2012;29(2):138-141. http://dx.doi.org/10.4067/ S0716-10182012000200003

9. Kim JD, Kim SY, Kwak EJ, Sol IS, Kim MJ, Kim KW, et al. Reduction rate of specific IgE level as a predictor of persistent egg allergy in children. Allergy Asthma Immunol Res. 2019:11(4):498-507. https://doi.org/10.4168/aair.2019.11.4.498

10. Boyano Martínez T, García-Ara C, Díaz-Peña JM, Muñoz FM, García Sánchez G, Esteban MM .Validity of specific IgE antibodies in children with egg allergy. Clin Exp Allergy. 2001;31(9):1464-1469. https://doi. org/10.1046/j.1365-2222.2001.01175.x

11. Perry TT, Matsui EC, Kay Conocer-Walker M, Wood RA. The relationship of allergen-specific IgE levels and oral food challenge outcome. J Allergy Clin Immunol. 2004;114(1):144-149. https://doi.org/10.1016/j.jaci.2004.04.009

12. Sampson HA. Utility of food-specific IgE concentrations in predicting symptomatic food allergy. J Allergy Clin Immunol. 2001;107(5):891-896. https://doi.org/10.1067/mai.2001.114708

13. Osterballe M, Bindsley-Jensen C. Threshold levels in food challenge and specific lgE in patients with egg allergy: is there a relationship? J Allergy Clin Immunol. 2003;112(1):196-201. https://doi.org/10.1067/mai.2003.1603

14. Huang F, Nowak-Wegrzyn A. Extensively heated milk and egg as oral immunotherapy. Curr Opin Allergy Clin Immunol. 2012;12(3):283-292. https://doi.org/10.1097/ $\mathrm{ACl} .0 \mathrm{~b} 013 \mathrm{e} 3283535 \mathrm{bc} 3$

15. Ando H, Movérare R, Kondo Y, Tsuge I, Tanaka A, Borres MP, et al. Utility of ovomucoid-specific IgE concentrations in predicting symptomatic egg allergy. J Allergy Clin Immunol. 2008;122(3):583-588. https://doi.org/10.1016/j. jaci.2008.06.016

16. Vazquez-Ortiz $M$, Pascal $M$, Jiménez-Feijoo R, Lozano J, Giner MT, Alsina L, et al. Ovalbumin-specific IgE/lgG4 ratio might improve the prediction of cooked and uncooked egg tolerance development in egg-allergic children. Clin Exp Allergy. 2014;44(4):579-588. https://doi.org/10.1111/cea.12273 